\title{
FINAL STAGE OF MUON BEAM COOLING
}

\author{
G. I. Silvestrov, A. N. Skrinsky, T. A. Vsevolozhskaya,BINP, Novosibirsk, Russia
}

\begin{abstract}
We consider the final stage of ionization cooling of muons, realized with the use of bent current-carrying lithiumberyllium rods, providing an optimum correlation between the cooling rates in all tree directions and minimum value of 6-dimensional emittance. The matching of lattice functions in a rod with those in accelerator structure is considered.
\end{abstract}

\section{INTRODUCTION}

The final stage of ionization cooling is characterized with reasonably low 6-emittance of muon bunches, achievable by very small magnitudes of betatron functions of the beam in slowing medium. Meanwhile the relative energy spread in a beam is not small, being equal to several percents even by optimum distribution of summary decrement between transverse and longitudinal degrees of freedom. This creates a problem of minimization of chromatic aberration effect by matching the low-beta focus in slowing medium with focusing structure of rather long-wave accelerator units.

\section{TRANSFER OF COOLING RATE TO LONGITUDINAL DIRECTION}

Bent current-carrying lithium-beryllium rods join in itself the functions of efficient focus and of degrader, necessary for transverse ionization cooling, and create a dispersion function, providing the longitudinal cooling - when correlated with transverse gradient of electron density in slowing matter - on account of redistribution of cooling decrement between transverse and longitudinal directions. The portion of decrement, transferred to longitudinal direction, is defined as $\Delta \delta=\delta_{0} \psi\left(\eta+\frac{1}{R}\right)$. Here $\delta_{0}$ is transverse decrement, equal to ratio $\xi / p v$, with $\xi$ standing for mean rate of ionization loss of energy, $\xi=-\frac{\overline{d E}}{d z} ; \psi$ denotes dispersion function, $\eta=\frac{1}{n_{e}} \frac{d n_{e}}{d r}$ - relative value of electron density gradient, and $R$ is the bend radius. Full expression for longitudinal decrement reads:

$$
\delta_{\|}=\xi^{\prime}+\delta_{0} \psi\left(\eta+\frac{1}{R}\right)
$$

where $\xi^{\prime}$ is a derivative of mean rate of ionization loss with respect to particle energy. It represents the natural longitudinal decrement. In energy region of logarithmic grow of loss rate the value of $\xi^{\prime}$ hardly achieves $7 \%$ of transverse decrement, while below $p c \sim 400 \mathrm{Mev}$, where it is negative, its absolute value $\left|\xi^{\prime}\right| \cong 2\left(\frac{1}{\gamma^{2}}-\frac{1}{L_{\mathrm{i}}}\right)\left(L_{\mathrm{i}} \sim 25 \div 30\right.$ is the logarithmic factor in expression for mean rate of ionization loss of energy) is growing fast with energy decrease and becomes equal to $\sim 0.9 \delta_{0}$ at momentum $\sim 100 \mathrm{MeV} / \mathrm{c}$. Thus, the energy of cooling is defined by a magnitude of product $\psi \eta$ we can create.

We consider two ways of creation of electron density gradient in current- carrying rods. In first (see fig. 1 a) the rod is composed of wedge-shape parts of two different metals [1]. One is the lithium, and another - the beryllium alloy with the same electrical conductivity as lithium. The relative gradient of electron density in such rod is found as: $\eta \cong \frac{1}{r_{0}} \frac{n_{e, B e}-n_{e, L i}}{n_{e, B e}+n_{e, L i}}$, where $r_{0}$ is a rod radius. Dispersion function in bent rod is equal to $\psi=r_{0} \frac{H_{0}}{H_{m}}$, where $H_{0}$ is bending field and $H_{m}$ - the focusing one, defined as $H_{m}=r_{0} \frac{d H}{d r}$. By equal each other magnitudes of above fields the value of $\psi$ is simply equal to $r_{0}$, and product $\eta \psi-$ to $\eta \psi=\frac{n_{e, B e}-n_{e, L i}}{n_{e, B e}+n_{e, L i}}$, that is to $\sim 0.56$. The ratio
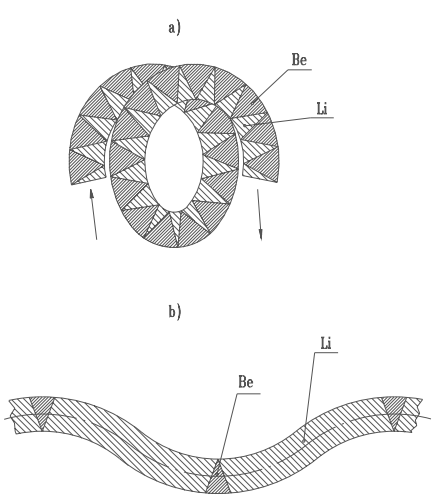

Figure 1: Two geometries of bent lithium-beryllium current-carrying rod.

$\psi / R=r_{0} / R$ adds to this a value of the order of 0.1 , so the longitudinal decrement is equal to $\delta_{\|} \cong \xi^{\prime}+0.66 \delta_{0}$. This provides the positive value of longitudinal decrement by beam momentum above $140 \mathrm{Mev} / \mathrm{c}$.

Another way of creation of electron density gradient consists in insertion of thin beryllium wedges between sections of lithium carrent-carrying rods (see fig. $1 \mathrm{~b}$ ). Wedge length $\Delta l$ (by the bottom) can not exceed $\sim 0.2$ of lithium section length $l$ without sufficient violation of focusing structure, which restricts an effective $\eta$ by a value: $\eta_{\text {eff }} \cong \frac{1}{r_{0}} \frac{n_{e_{, B e}} \Delta l}{2 n_{e, L i} l+n_{e, B e} \Delta l} \leq 0.26$. This is sufficiently less the value got in first case, however this reduction can be compensated by means of enlarging of dispersion function magnitude at wedges. This is achieved by a special configuration of bent rod, providing the parallel shift of a beam between the wedges. With phase advance of free particle 
oscillations between two subsequent wedges equal to $220^{\circ}$ $-240^{\circ}$, the absolute value of $\psi$ at wedges is increased by $3-3.5$ times. This permits to get the product $\psi \eta$ equal to $\sim 0.8 \div 0.9$.

\section{ULTIMATE VALUE OF NORMALIZED 6-EMITTANCE}

An optimum relation between the transverse and longitudinal decrements by cooling in current-carrying rods with restricted value of maximum field at the rod surface, defines the longitudinal decrement as equal to one fourth of the sum decrement of ionization cooling $\delta_{\Sigma}=2 \delta_{0}+\xi^{\prime}$, i.e. $\delta^{\text {(opt })}=\delta_{0} / 2+\xi^{\prime} / 4$.

In the wedge-rod scheme the optimum value of $\delta_{\|}$is achieved with $\Delta l / l \cong 0.13$ by cooling at $200 \mathrm{MeV} / \mathrm{c} \mathrm{mo-}$ mentum.

Dependence of an ultimate value of normalized 6dimensional emittance on energy is defined by a factor $\sim \frac{\left(\gamma^{2}+1\right) \sqrt{\gamma}}{\beta^{2}}$ [2]. It is minimum at $p c \cong 100 \mathrm{MeV} / c$, where the value of $\epsilon_{\mathrm{eq}, \mathrm{N}}^{(6)}$ is estimated as:

$$
\epsilon_{\mathrm{ult}, \mathrm{N}}^{(6)} \sim \cdot 10^{-4} \frac{1}{H_{m}^{2}} \sqrt{\frac{\lambda}{2 \pi e \varepsilon}} \mathrm{cm}^{3}
$$

in dependence on maximum focusing field $H_{\mathrm{m}}$, acceleration rate $e \varepsilon$, and wave length of accelerating voltage $\lambda$. With $H_{m}=10 \mathrm{~T}, \lambda=10 \mathrm{~cm}$, and $e \varepsilon_{\text {eff }} \cong 1.2 \mathrm{MeV} / \mathrm{cm}$ one gets: $\epsilon_{\mathrm{ult}, \mathrm{N}}^{(6)} \sim 1.2 \cdot 10^{-6} \mathrm{~cm}^{3}$.

At particle momentum $200 \mathrm{MeV} / c$ and with more moderate accelerator parameters $-\lambda \cong 30 \mathrm{~cm}$ and $e \varepsilon_{\text {eff }} \cong$ $0.5 \mathrm{MeV} / \mathrm{cm}-$ this value is: $\epsilon_{\mathrm{ult}, \mathrm{N}}^{(6)} \sim 4.0 \cdot 10^{-6} \mathrm{~cm}^{3}$.

Cooling of $\sim 200 \mathrm{MeV} / \mathrm{c}$ momentum muons in a system, composed of bent lithium rods and beryllium wedges of 0.13 relative length, is illustrated in figure 2 .

Curves 1 and 2 show, accordingly, the radial and axial normalized emittances in $\mathrm{MeV} / \mathrm{c} \mathrm{cm}$ and 3 - the longitudinal emittance in $\mathrm{MeV} \mathrm{cm}$ versus the number of degraderaccelerator cells. Thin lines present the numerical solution with the use of kinetic equation, thick ones - the result of simulation with Moliere formulae, used for the Coulomb scattering angle distribution, and Vavilov formulae - for straggling of energy loss.

Dashed line shows the ratio $R_{t}$ of rod radius to maximum r.m.s. particle coordinate, got by the numerical solution, while dashed squares at figure bottom show the particles (from 100 initial), found lost by simulation. Both dipole and maximum focusing fields are taken equal to 10T, acceleration rate - to $0.5 \mathrm{MeV} / \mathrm{cm}$, and $\lambda-$ to $30 \mathrm{~cm}$. The initial r.m.s. momentum spread is $\pm 4.5 \%$ and longitudinal coordinate $- \pm 1 \mathrm{~cm}$.

The rod radius is gradually reduced with beam cooling from $\sim 8 \mathrm{~mm}$ down to $\sim 3.5 \mathrm{~mm}$, which provides with almost constant value of $R_{t}$, equal to $\sim 2.5\left(r_{0} \cong 3.5 \sigma_{\perp}\right)$. The rod length is also decreased in proportionality with $\sqrt{r}_{0}$ to keep constant value $\nu \varphi_{0}=\frac{2}{3} \pi$. The energy loss

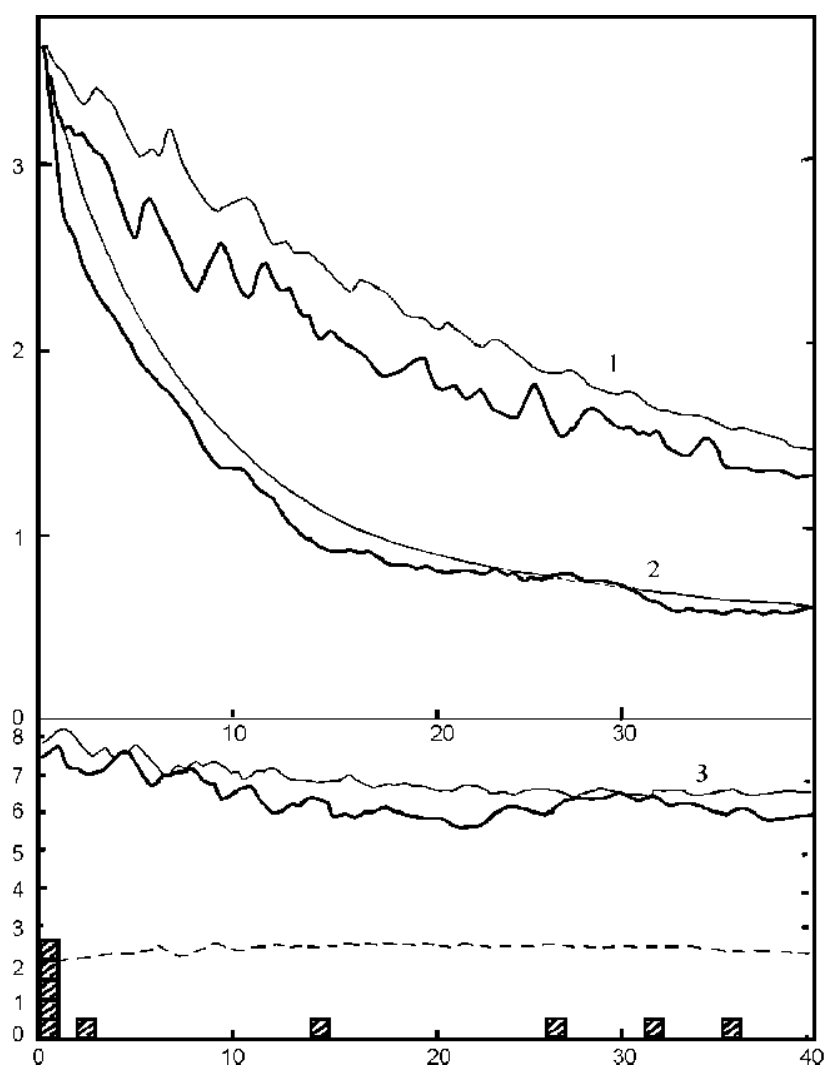

Figure 2: Cooling of $200 \mathrm{MeV} / \mathrm{c}$ muons.

per cell is about $30 \mathrm{MeV}$ in the beginning and about 20 $\mathrm{MeV}$ in the end.

The final radial emittance is more than two times larger than the axial one, which proves, that more than half of radial decrement is transferred to the longitudinal direction. The initial longitudinal emittance is taken close to the equilibrium value and thus only slightly decreases with cooling.

The magnitude of normalized 6-dimensional equilibrium emittance, got in the end of 40-cell cooling, is equal to $4.5 \cdot 10^{-6} \mathrm{~cm}^{3}$ in good accordance with analytic estimation.

\section{COMPENSATION OF LINEAR CHROMATICITY BY ACCELERATION}

To get the defined above ultimately small normalized 6emittance of muon beam there has to be solved a problem of minimization of chromaticity of focusing by acceleration between the degrading sections being the necessary part of ionization cooling scenario.

Such minimization is achieved by compensation of linear component of chromaticity, as it is considered below.

The transverse mean square beam characteristics by acceleration are defined with equations:

$$
\begin{aligned}
& \frac{\partial\left\langle r^{2}\right\rangle}{\partial y}-2 \frac{p\langle r \theta\rangle}{e \varepsilon_{0}}=0 \\
& \frac{\partial p\langle r \theta\rangle}{\partial y}-\frac{p^{2}\left\langle\theta^{2}\right\rangle}{e \varepsilon_{0}}+\frac{k_{\text {eff }} p^{2}\left\langle r^{2}\right\rangle}{e \varepsilon_{0}}=0
\end{aligned}
$$




$$
\frac{\partial p^{2}\left\langle\theta^{2}\right\rangle}{\partial y}+2 \frac{k_{\text {eff }} p^{3}\langle r \theta\rangle}{e \varepsilon_{0}}=0 .
$$

Here $r$ and $\theta$ are 2-vectors of transverse coordinate and angle, accordingly, $y$ stands for particle rapidity $y=\ln \frac{E+p}{M}$, $E$ and $p$ are energy and momentum, and velocity of light is put equal to $1 ; e \varepsilon_{0}$ denotes the acceleration rate, and $k_{\text {eff }}-$ the focusing strength, defined by means of derivative of angle with respect to longitudinal coordinate as $k_{\text {eff }} r=-\frac{d \theta}{d z}$.

Let us consider the focusing strength to be of quadratic dependence on particle momentum, $k_{\text {eff }} \propto 1 / p^{2}$, and about uniformly distributed through the length of acceleration. Such a focusing can be really created with the use of continuous train of short quadrupoles ${ }^{1}$ with accelerator, placed in their aperture (see fig. 3). The focal distance of short FD pair $f$ is about equal to $\frac{1}{k^{2} l^{3}}$ with $k=e \frac{d H}{d r} / p c$ and $l$ being a length of quadrupole unit. The effective value of $\frac{d \theta}{d z}$ is thus equal to $\sim \frac{r}{2 l f}$, and $k_{\text {eff }} \cong k^{2} l^{2} / 2$.

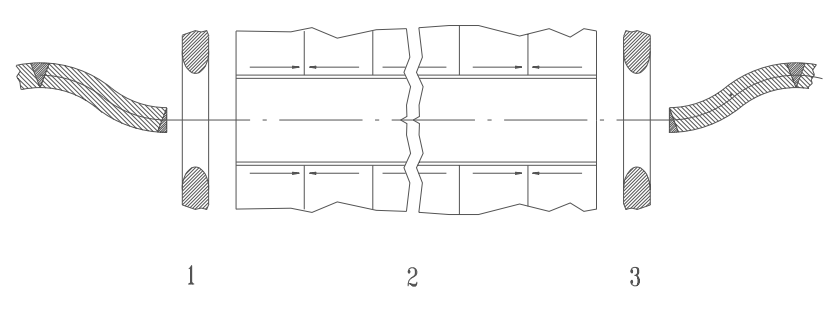

$1,3-$-short solenoidal lenses with field up to $25 \mathrm{~T}$; 2-quadrupole (helic) tract.

Figure 3: Scheme of beam focus between degraders.

Solution of system (1) reads:

$$
\begin{aligned}
& \left\langle r^{2}\right\rangle=\left\langle r_{0}^{2}\right\rangle \cos ^{2} \omega\left(y-y_{0}\right)+\frac{\left\langle\theta_{0}^{2}\right\rangle p_{0}^{2}}{\kappa} \sin ^{2} \omega\left(y-y_{0}\right) \\
& \langle r \theta\rangle p=\frac{\left\langle\theta_{0}^{2}\right\rangle p_{0}^{2}-\kappa\left\langle r_{0}^{2}\right\rangle}{2 \sqrt{\kappa}} \sin 2 \omega\left(y-y_{0}\right) \\
& \left\langle\theta^{2}\right\rangle p^{2}=\left\langle\theta_{0}^{2}\right\rangle p_{0}^{2} \cos ^{2} \omega\left(y-y_{0}\right)+\kappa\left\langle r_{0}^{2}\right\rangle \sin ^{2} \omega\left(y-y_{0}\right)
\end{aligned}
$$

with $\kappa=k_{\text {eff }} p^{2}$, and $\omega=\frac{\sqrt{\kappa}}{e \varepsilon_{0}}$. Here we neglected the value of $\left\langle r_{0} \theta_{0}\right\rangle$, which is small in current-carrying rod.

To get a small betatron function at entrance to next lithium rod the phase advance $\omega\left(y-y_{0}\right)$ is to be chosen equal to $\pi$ (or $2 \pi$ and so on), which defines the necessary value of $\kappa$ by fixed acceleration rate. On acceleration from $\sim 170$ up to $200 \mathrm{MeV} / \mathrm{c}\left(y-y_{0} \cong 0.14\right)$ with phase advance equal $\pi$ and acceleration rate $e \varepsilon_{0}$ to $\sim 0.5 \mathrm{MeV} / \mathrm{cm}$, the value of $\kappa$ is $\sim 126 \mathrm{MeV}^{2} / \mathrm{c}^{2} \mathrm{~cm}^{2}$.

The magnitudes $\left\langle r^{2}\right\rangle$ and $\left\langle\theta^{2}\right\rangle$ at accelerator exit in linear approach do not depend on particle energy deviation. Aberrational effect manifests itself only through $\langle r \theta\rangle$. It is proportional to a difference $\left\langle\theta_{0}^{2}\right\rangle p_{0}^{2}-\kappa\left\langle r_{0}^{2}\right\rangle$, which is not

\footnotetext{
${ }^{1}$ Technologically preferable looks the helical quadrupole tract
}

small as far as $\kappa$ is much less than corresponding magnitude in a rod: $\kappa<<p_{0}^{2} / \beta_{0}^{2}$. In result, the value of $\delta\langle r \theta\rangle$ at accelerator exit is rather large. In following lithium rod it gives rise to oscillating addition to betatron function:

$$
\delta \beta_{0} \cong-\frac{\delta E}{e \varepsilon_{0}} \frac{p_{0}}{p}\left(1-\frac{p_{0}}{p}\right) \sin 2 \sqrt{k_{0}} z
$$

with amplitude, being of the order of or even exceeding the value of $\beta_{0}$ by $\delta p / p$ equal to several percents.

This makes evident the necessity of matching lens to be inserted between the lithium rod and accelerator. Such a lens, making the $\pi / 2$ transformation with focal distance $f_{0} \cong \sqrt{\frac{\beta_{0} p_{0}}{\sqrt{\kappa}}}$, will provide zero value of $\langle r \theta\rangle$ by acceleration. Simultaneously a lens with focal distance $f \cong$ $\sqrt{\beta_{0} p \sqrt{\frac{p}{p_{0} \kappa}}}$, put after accelerator, will match the beam with focus in next rod.

Thus the linear chromaticity of focusing by acceleration is compensated. This, however, does not mean the compensation of chromatic aberration, but the trajectory of aberrational displacement of particle coincides in lenear approach with transverse phase trajectory.

The matching lenses also make contribution to the aberrational effect. With account for this made, the necessary value of $f_{0}$ is defined as:

$$
f_{0} \cong \sqrt{\frac{p_{0}}{p}}\left\{\frac{\beta_{0}^{2} p_{0}\left(p-p_{0}\right)}{\nu e \varepsilon_{0} E} /\left[\frac{E_{0}}{E}+\left(\frac{p_{0}}{p}\right)^{7 / 4}\right]\right\}^{1 / 3},
$$

where $\nu$ stands for a power of matching lens focal distance dependence on particle momentum: $f_{0} \propto p^{\nu}$. The value of $f$ is found as $f=f_{0}\left(\frac{p}{p_{0}}\right)^{3 / 4}$.

By $p_{0}=170 \mathrm{MeV} / \mathrm{c}$ and $p=200 \mathrm{MeV} / \mathrm{c}, e \varepsilon_{0}=0.5$ $\mathrm{MeV} / \mathrm{cm}, \beta_{0}=2 \mathrm{~cm}$ and $\nu=2$ the magnitudes of $f_{0}$ and $f$ are equal to 4.3 and $5.4 \mathrm{~cm}$, accordingly.

The lens with such a focal distance for particles of $\sim 200$ $\mathrm{MeV} / \mathrm{c}$ momentum is a problem. It can not be the lithium one because with beta-function of beam, exceeding $10 \mathrm{~cm}$, the multiple scattering in lithium will result in beam emittance increase by $\sim 10 \%$ of equilibrium value in each lens.

The only solution seems to be a short solenoidal lens with very high - up to $25 \mathrm{~T}$ - pulsed magnetic field.

The use of "linear" systems ( $k_{\text {eff }} \propto 1 / p$ ) for beam focusing by acceleration would evidently give an advantage in aberrational effect as compared to the "quadratic" ones. As such a "linear"systems the plasma lenses can be considered. Having the focal distance linearly dependent on particle momentum, such lenses by rather moderate parameters would provide an efficient focusing with minimum chromatic aberration.

\section{REFERENCES}

[1] . Skrinsky, AIP Conference Proceedings 441, p. 249, (1997).

[2] . A. Vsevolozhskaya, Proc. of EPAC'98 NIM A, being published. 